\title{
José Aburto Zolezzi: el autor en busca de la interfaz literaria ideal
}

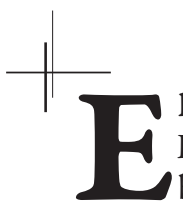

1 materialismo textual, interfaces literarias y José Aburto Zolezzi

Hay autores a quienes unas palabras "simples" (es decir, impresas en unas páginas blancas con tinta negra) no parecen medios suficientes para la comunicación literaria. Ellos buscan maneras de emplear el código verbal distintas de las más comúnes y populares. Pero si estos autores huyen de lo tradicional no lo hacen por aburrimiento, sino buscando nuevas formas de expresión y creación literaria. José Aburto Zolezzi, a quien vamos a presentar como uno de estos artistas, explica: "Lo que mejor define mi trabajo es la constante experimentación en formatos, soportes y métodos de escritura que reinterpretan el quehacer poético desde una perspectiva propia: interactiva, tecnológica y personal" (Aburto Zolezzi s. a. b). El autor añade que su trabajo "ha estado dedicado a explorar las posibilidades del mundo digital y su impacto en las formas de expresión” (Aburto Zolezzi s. a. b). Buscando estos nuevos modos de comunicación literaria Aburto Zolezzi no se limita a la literatura electrónica, sino es también autor de unos textos “analógicos" (impresos o al menos "hechos" en papel), instalaciones literarias en los espacios reales y unas obras que unen los medios electrónicos con otros. Así, su obra entra en el intrigante campo de la literatura moderna que nace del diálogo entre la forma tradicional del libro y sus diversas formas electrónicas, el diálogo del que nacieron interesantes libros impresos, aunque "extraños" y atípicos, que hoy en día podemos encontrar en las librerías (entre los más discutidos se pueden enumerar todas las obras de Mark Danielewski, Raw Shark Text de Steven Hall, La gente de papel de Salvador Plascencia o La invención de Hugo Cabret de Brian Selznick ${ }^{1}$ ).

$1 \quad$ Vale la pena mencionar aquí también los libros augmentados (AR-books) como Notebook de Tomasz Lipko Between Page and Screen de Amaranth Borsuk y Brad Bouse (sin recordar muchas publicaciones de este tipo dedicadas a los niños). 
Por eso vamos a analizar la obra de Aburto Zolezzi en el contexto del denominado materialismo textual (textual materialism), un pensamiento dedicado a los "textos encarnados" 2 (Starre 2015: 44), que une diversas teorías que abandonan la visión de la comunicación literaria basada solamente en el código verbal ${ }^{3}$. Según esas teorías, no todo texto es un mensaje (verbal) que se puede verter como un líquido de un recipiente al otro, algunos son inseparables de su forma material y su sentido no consiste solamente en las palabras. Estos son textos que tienen sus "cuerpos", las obras literarias con las interfaces no invisibles ${ }^{4}$. Muchos investigadores llevan años trabajando en este campo, desarrollando conceptos y teorías que con diversos nombres describen el mismo fenómeno. Así se puede aludir a por ejemplo: Espen Aarseth, con su idea de la literatura ergódica (Aarseth 1997), Katherine Hayles, con su idea de technotextos (Hayles 2002), Johana Drucker, que caracteriza diversas interfaces literarias (Drucker 2011), Lori Emerson, que escribe de reading/writing de las interfacez literarias (Emerson 2014), Glyn White, que trata de graphic surface (White 2005), Alexander Starre, con su concepto de metamedia (Starre 2015) o Jessica Pressman, que desarrolla teoría de bookishness (Pressman 2009). En el contexto del análisis que vamos a presentar en este artículo entran también trabajos como despliegues de teoría de la liberatura o aspectos liberarios de la literatura (Przybyszewska 2015; Bazarnik 2016) o, en general, varios estudios de las plataformas literarias (literary platform studies) (por ejemplo, Marecki 2018).

En las teorías que nos interesan se señala frecuentemente que no tiene importancia el medio en que está realizada la obra, porque el concepto de hacer la interfaz semánticamente importante, visible y perceptible resulta totalmente transmedial ${ }^{5}$. Por eso algunos teóricos se concentran en mostrar similitudes entre lo analógico y lo electrónico. Estos aspectos los vamos a ilustrar con el análisis de la obra de Aburto Zolezzi, un autor que crea no solo la literatura analógica ("de papel"), sino también la electrónica y las obras, probablemente más interesantes, que unen dichos dos campos.

En este artículo vamos a analizar algunos trabajos de Aburto Zolezi para ver qué significan en realidad sus declaraciones mencionadas, verificando al mismo tiempo las metodologías del materialismo textual. El término clave para esto será "la interfaz literaria", cada día más popular en el pensamiento humanístico y crucial para las teorías que hemos citado. Los análisis que vamos a presentar servirán como ejemplo de lo que significa "leer la interfaz" de la obra literaria (acción que, según las teorías a las que hemos aludido, tiene que realizar el lector para entenderla) y "buscar una interfaz ideal para cada historia" (una frase que, en nuestra opinión, caracteriza perfectamente la idea de la creación artística de Aburto Zolezzi).

2 El término inglés dice: "embodied text".

3 Así nuestro artículo va a presentar media specific analysis.

4 La forma traditional del libro la podemos ver como una interfaz invisible, porque - como lo señalaba Josef Bezos (Levy 2007) - este "desaparece" en las manos del lector, quien pierde la noción de su existencia. En tal caso, el libro sirve solo como un medio útil, práctico y cómodo para transmitir el mensaje de la obra.

5 Lo observamos perfectamente en una serie de obras que emplean diversos medios para despertar cierta sensación en el lector, como Lluvia de Leopold Staff (un texto que nos hace oír sonidos como si fuera lluvia), el caligrama Lluvia de Apollinaire (un poema con versos dispuestos en forma de lluvia) y Text Rain de Romy Achituv y Camille Utterback (un texto que actúa como la lluvia, un poema que deja caer sobre el lector una lluvia de letras). 
De acuerdo con los teóricos mencionados, Aburto Zolezzi señala que "el texto no está circunscrito en el concepto o en la idea que se quiere transmitir, sino en el uso del lenguaje que se ha hecho para transmitir ese concepto o idea" ${ }^{6}$ y busca para sus obras las formas que servirán de la mejor manera posible a realizar sus planes artísticos. Por eso el poeta frecuentemente sobrepasa los límites de los medios y rompe totalmente con los hábitos de lectura. Sus trabajos absorben a los lectores de diversas maneras y apelan, no metafóricamente, a todos los sentidos. En consecuencia, sus obras no solo son visibles, sino frecuentemente audibles o incluso táctiles. Son textos para experimentar, para leer con el cuerpo y los sentidos. Estos aspectos corporales de la comunicación literaria, la aplicación de los gestos del lector o sus movimentos (o al menos su presencia en el espacio real) en el acto de leer será uno de los aspectos más importantes que vamos a analizar en la obra de Aburto Zolezzi.

\section{Que yo cometa: un poema para tocar que nos "muestra" su dolorosa huella del pasado}

Que yo cometa es un texto que, para subrayar su forma no-electrónica, podemos llamar "analógico"; es un poema escrito a mano y recortado en papel. Es decir, sus versos tienen forma de un recorte artístico de papel, un recorte hecho de letras y palabras (ilustración 1a). Gracias a esta interfaz literaria el lector puede "sacar" parte del texto del poema, modificándolo. De este modo, la forma de la obra elegida por Aburto Zolezzi le permite al texto ser interactivo de manera típica para la literatura electrónica. Que yo cometa parece abierto a "ser leído con un gesto" del lector, abierto a un acto de "grabar las palabras" por él (en este caso - literalmente), así como Janez Strehovec describe los actos de e-lectura (Strehovec 2014: 354). Así, este poema "analógico" entra en el campo de la reflexión sobre los aspectos táctiles, hápticos (en general, corporales) de una lectura, reflexión orientada no solo a unos textos e-literarios para leer en la pantalla de un ordenador personal o en unas instalaciones e-literarias interactivas, sino también dirigida a obras, cada día más populares, dedicadas a pantallas táctiles (Ensslin 2011; Gibs y Angel 2013). Aburto Zolezzi muestra con su obra que no es la forma del texto, ya sea analógica o electrónica, lo que decide de su interactividad o de la posibilidad de implementar los aspectos hápticos, sino la manera ideada de leer la obra que la hace interactiva e invita al lector a leerla con todos sus sentidos.

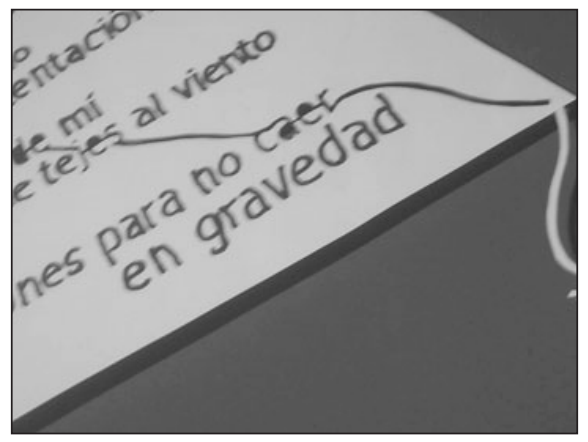

Ilustración 1a.

6 Artículo citado lleva un subtítulo significativo y lleno de referencias: “¿El soporte determinará el contenido?”. 
En general, Que yo cometa trata de la separación de unos amantes, el “yo" lírico se concentra en el dolor que le hace sufrir la situación. En la primera lectura obtenemos un texto que sugiere que el sujeto lírico acepta lo que ocurre, que deja a su amor irse ${ }^{7}$; parece que el "yo" se siente preparado/a a estar solo/a y olvidar, a cerrar una etapa de su vida, o al menos declara que sabe vivir sin el amor pasado. Sugiere que solo necesitaría que este (como dice el texto) "llévese" de él y "líberele" de su influencia.

Sin embargo, luego viene lo que podemos llamar la segunda lectura. Si sacamos la parte recortada del texto (la que por su forma nos pide hacerlo), obtenemos un texto modificado. Este es al mismo tiempo un poco más corto y más largo del inicial: las palabras sacadas del cuerpo del texto forman ahora su punto culminante, un verso adicional que dice: "pero no me dejes caer”. Estas palabras indican que en realidad el sujeto lírico no se imagina vivir sin el amor pasado, que la separación no es tan fácil como él/ella esperaba. Lo entendemos de diversos modos. Primero, lo dice el texto nuevo, en el cual la falta de palabras recortadas cambia el sentido original. Eso se nota perfectamente con los versos iniciales del poema: en vez de „Espero que te vayas / y no dejas nada tras de ti que no haya sido mío antes” leemos “Es que te vayas / y no dejas nada tras de ti que haya sido mío antes”. En segundo lugar, lo notamos con el marco que ha dejado el propio texto al recortar parte de él. Vemos el texto nuevo, pero este lleva una huella, una señal evidente de lo ocurrido. Es un rastro que no se puede borrar, que permanece en el texto, que se queda para siempre como parte de él. Además, esa huella tiene forma de un agujero, pues, en otras palabras, lo que vemos es el vacío, la ausencia que sufre el “yo" lírico.

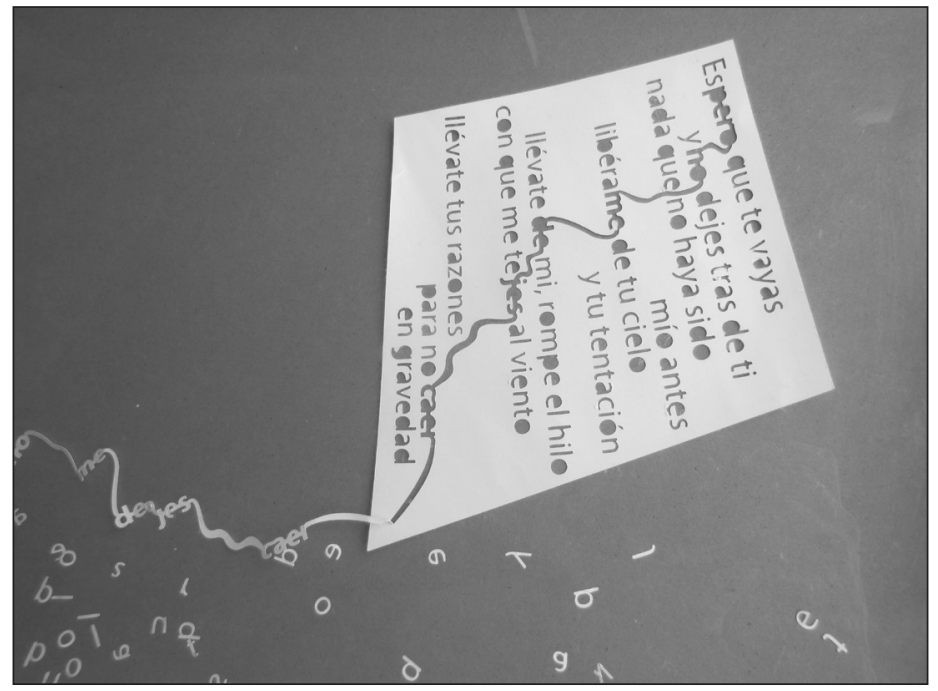

Ilustración $1 b$.

7 Se puede también interpretar el texto como la imagen de los miedos del sujeto lírico: aunque nada ha pasado, él tiene miedo de la separación, que significaría falta de confianza en sí mismo o en la relación. 
De este modo, la forma material del texto refleja el contenido de la obra. El amor pasado nos deja marcados para siempre, es un vestigio que nos construye y cuya influencia no podemos negar. Por eso el sujeto lírico, tirado por emociones opuestas, está en conflicto. Y ese conflicto está reflejado en la forma del texto - el fragmento ya ausente, "extirpado" del texto inicial, deja en el poema una huella, una ausencia palpable. Entonces la interfaz del texto elegida por el autor parece ideal para comunicar la complejidad de la situación lírica que acabamos de describir ${ }^{8}$.

$\mathrm{Al}$ mismo tiempo, esta interfaz entra en el diálogo con la tradición literaria y esto lo logra aun siendo única, inventada para una obra y un mensaje concretos. Aburto Zolezzi creó un texto que se refiere a la tradición de la poesía visual (diversos poemas con intextos, como por ejemplo, carmina cancellata o imago poem); con la única diferencia de que su obra es más táctil e interactiva que las formas mencionadas. Asimismo, Que yo cometa está muy cerca de la tradición de la literatura permutativa y combinatoria (no solo electrónica) ${ }^{9}$, un tipo de literatura cuya lectura permite diversas permutaciones del texto y que también tiene su larga historia (Higgins 1987; Rypson 1989, 2002; Rettberg 2019). Una estrecha relación con la historia del arte (no solamente literario) y sus formas es muy típica de las obras de Aburto Zolezzi — al autor le gusta jugar con la tradición y las normas del arte. Sin embargo, jugar no es su único objetivo, el juego encierra un profundo sentido, es un elemento del programa poético orientado a la "constante" (ya que está vinculada a la tradición) "experimentación en formatos, soportes y métodos de escritura que reinterpretan quehacer poético" (Aburto Zolezzi, s. a. c) o literario en general. Es la búsqueda de una interfaz ideal para lo que el poeta quiere transmitir.

\section{Grita: un poema que nos "obliga" a gritar para poder leerlo}

En cuanto al campo de la literatura electrónica, Aburto Zolezzi también crea textos en los cuales la conexión entre la forma del texto (interfaz elegida por el autor para la comunicación literaria) y el contenido de la obra parece crucial. En este artículo vamos a analizar solo dos de los numerosos textos electrónicos del autor. El primero será el poema Grita del ciclo Pequeñas interfaces poéticas - el fin del click. Cada uno de los tres textos del ciclo está presentado en la pantalla, que suele ser la solución más típica para las obras electrónicas. Sin embargo, para manejarlos no recurrimos a ninguno de los otros elementos de interfaces clásicas como un teclado, ratón o una simple palanca de juego. En su lugar, encontramos unos cubos de colores de los que cada uno contiene un elemento que sirve para empezar la lectura y permite continuarla.

8 Hay también otra versión del poema (ilustración 1b), en la cual también algunas otras letras del texto están en parte recortadas (permanecen una parte del texto principal, se le puede reconocer y leer pero se nota que al texto le ha pasado algo). De este modo el poema muestra, que nuestra visión de lo pasado siempre se cambia un poco con el transcurso del tiempo. Así a esta obra material la podemos ver también como un texto que trata de la memoria y del proceso de olvidar.

9 Las permutaciones y cambios visibles del texto son medios estilísticos frecuentemente usados por el poeta, típicos para él. Los usa desde hace años, entre ejemplos se puede mencionar el poema no-electrónico (un poema-evento) Des(p)ierto (2006) en el que las letras desaparecen (por culpa del agua), modificando el sentido. El poeta mismo define este tipo de escritura como "la técnica de «descritura»" (Aburto Zolezzi s. a. d). También en los ciclos Pequeñas Interfaces Poéticas y Matters. Electromagneticpoems (a los cuales pertenecen e-poemas Grita y El papel líquido analizados en este artículo) encontramos interesantes ejemplos de las obras combinatorias: los textos Concepción del Dragón (un poema que se desarrolla y evoluciona hasta llegar a su forma final gracias a la actividad física del lector) y Solid Blot (en el que el punto que "camina" por el texto lo va modificando). 
En el caso de Grita hay un micrófono escondido dentro de un cubo rojo. ¿Para qué sirve? La invitación a gritar puesta en el título de la obra es auténtica — para leer este poema hay que gritar, o, mejor dicho, no se puede leerlo sin gritar; es un texto al que tenemos que levantar la voz para que se muestre en la pantalla. Además, si dejamos de gritar, el texto desaparece y lo único que nos queda, si queremos conocerlo entero, es volver a empezar la lectura gritando sin pausas. He aquí que surge otra pregunta: ¿qué sentido tiene este juego? ¿Qué sentido tiene gritar al texto? Y parece ser una pregunta razonable, dado que no solo es incómodo leer gritando, sino también gritar leyendo. Si de esta manera Aburto Zolezzi rompe totalmente con nuestros hábitos de lectura, no lo hace solamente para jugar.

Cuando el grito resulta suficientemente espaciado (es más fácil realizarlo en grupo ${ }^{10}$ ), en la pantalla aparece un texto que tiene mucho que ver con lo que el lector está haciendo. Es por ello que el monólogo lírico trata del esfuerzo, esfuerzo también físico, que el autor pone en el proceso de crear la obra. El poema viene de los "adentros" del poeta, del "huracán" que lleva dentro. Escribir, según el "yo" lírico, es como gritar hasta perder fuerzas, gritar con todo su cuerpo y su alma. Y como lo señala el verso final del poema, esta voz, este grito fuerte de los adentros, le "guía" al artista. Lo que le permite al lector experimentar mejor el esfuerzo del poeta es imitarlo. Grita le permite sentir dicha sensación. Para poder finalizar su lectura, el lector tiene que gritar casi hasta perder las fuerzas o hasta que se le corte la respiración. No solo lee sobre cómo se siente el artísta durante el proceso de la creación, sino que él mismo siente cómo el poema va naciendo del dolor y del sufrimento físico, del grito (el texto dice, por ejemplo: "tirantes cuelguen de mis labios / las palabras reventadas de saliva"). Esta sensación, tan original e insólita de la lectura, basada en una repetición de las experiencias del "yo" lírico en el espacio y la vida real del lector, es posible tan solo gracias a la interfaz elegida por Aburto Zolezzi para este poema.

Dicha manera de imitar las sensaciones del "yo" del texto por el lector es algo muy típico de la literatura electrónica actual (María Ángel y Anna Gibbs describieron los "gestos digitales" que sirven para crear esta impresión). Serge Bouchardon no solo las usa frecuentemente en sus trabajos (por ejemplo, en Loss of Grasp o en Do It), sino también ha propuesto introducir a las poéticas unas nuevas figuras retóricas, las que usen gestos de lectores (Bouchardon 2014, 2018). Aburto Zolezzi emplea estas figuras en muchos de sus trabajos, mostrando que no son específicas únicamente para las obras hechas con uso de los nuevos medios. El autor demuestra que también se pueden aprovachar con éxito en la literatura no-electrónica.

\section{Extravia: hipertexto físico que nos "propone" ir caminando por la mente y seguir el pensamiento}

En general, a Aburto Zolezzi le gusta mostrar con sus obras que no solo las figuras retóricas sino también las formas y los géneros pensados como característicos para la literatura electrónica se pueden crear igualmente sin uso de ordenadores y pantallas. Lo podemos ver en Extravía, el "hipertexto físico" (Aburto Zolezzi, s. a. c), como lo describe el poeta mismo. Este trabajo es una instalación en forma de "pasadizos flotantes a la altura de la cabeza a manera de

10 Este hecho abre el tema de las posibilidades de leer en galerías y de los problemas vinculados a nuevos puestos de presentación de los textos electrónicos (y también de la lectura común). Este problema fue importante en el contexto de la poesía concreta y sigue vigente desde hace años en el contexto de textos e-literarios en forma de instalaciones que solo se pueden ver/leer en los espacios públicos, que rompen con la tradición de lectura individual, como los famosos Text Rain o Legible City). 
un laberinto" (Aburto Zolezzi, s. a.c). Las paredes de los mencionados pasadizos están hechas de placas rojas, cubiertas, pero no todas, con el texto escrito con tinta blanca. Así el trabajo toma forma de un texto no-linear, por el que el lector "navega" eligiendo su camino. Sin embargo, en este caso, lo elige literalmente y no con un simbólico "click" del ratón.

Obviamente, Extravía no es la única obra de este tipo en la historia de la literatura. Los hipertextos en los espacios reales los crearon, por ejemplo, Małgorzata Dawidek Gryglicka o Andrzej Bednarczyk ${ }^{11}$. Por otra parte, hay todo un contexto de la así llamada place-bound literature (Løvlie 2009): las narrativas locativas en amplio sentido ${ }^{12}$, los textos literarios (y no solo literarios) para "ser caminados" que no siempre usan lo electrónico ${ }^{13}$. Con su Extravía Aburto Zolezzi entabla también un diálogo con toda esta tradición.

Pero lo más interesante de su hipertexto físico parece el hecho de que no es un texto-laberinto "normal", "clásico" ( si en este contexto nos sirve una categoría como esta) ${ }^{14}$. Extravía, efectivamente, es un texto para caminar y para errar, pero es también un laberinto sin entrada ni salida. Y como en otros trabajos de Aburto Zolezzi este absurdo tiene su sentido. Para encontrarlo hay que pensar en el texto y la relación entre la manera de leerlo, ideada por el autor, y lo que se lee.

El texto de Extravía invita a sus lectores a olvidar las reglas: las de leer (de manera linear y sin moverse, olvidando su cuerpo) y las de caminar por laberintos (con la obligación de entrar y buscar cómo salir). La peregrinación literaria fue proyectada únicamente "para los que saben que solo agachando la cabeza se entra en razón / para ellos se ha delineado este ritual / este peregrinaje de vuelta a la primera duda" ${ }^{15}$. Hay que romper con todas las reglas, olvidar a lo que estamos acostumbrados, incluso para tan solo empezar la lectura. La forma del laberinto en el que entramos recuerda la del cerebro. Por lo tanto, los lectores, si realmente se atreven a agachar la cabeza para entrar en él, dado que es la única manera de hacerlo, recorren el laberinto del pensamiento. El texto anima a los lectores: "esta severa invitación engloba de rojo los sentidos y le promete seguridad", insiste en que "no tengan miedo de perder la cabeza que peor es perder el cuerpo del cuello para abajo". Invita a dejar de pensar en el mundo exterior (donde el lector se ve sin cabeza) y dejarse llevar por la corriente de palabras dentro del laberinto. Se puede interpretar Extravia como una forma material, "encarnación" de la corriente de la consciencia. Así el peregrinaje entre las frases cortadas (sin partes finales o iniciales) y no siempre lógicas, va cobrando sentido. Errar por el laberinto refleja la agitación de nuestra mente; tener que elegir el camino, decidir hacia dónde girar imita la corriente del pensamiento. Así, esta peregrinación nos permite experimentar una multititud de posibilidades y sentir las consecuencias de elegir solo una de ellas. Al mismo tiempo, es un viaje en que leemos sobre el proceso de pensar y crear, en el que caminando, acercándonos al centro, nos acercamos al entendendimiento. No obstante, para entender, tenemos que olvidarnos del mundo exterior, perderlo de vista y permanecer rodeados tan solo de aquellas paredes rojas

11 Se puede también pensar aquí en el famoso między (entre) de Stanisław Dróżdż.

12 Por ejemplo Jeremy Hight propone usar el término "narrativa locativa” como "umbrella term” (High 2010: 319 ).

13 Muy interesantes ejemplos nos aportan los trabajos del grupo Time's Up (por ejemplo: Turnton Docklands), caracterizados por ellos mismos como "physical narratives" (es decir: las narrativas físicas/materiales).

14 No tenemos espacio para desarrollar aquí esta hipótesis, pero Aburto Zolezzi también dialoga con diversos conceptos del laberinto.

15 Para poder citar algunos fragmentos del texto uso el mapa facilitado por el autor. La manera de escribirlos refleja su disposición en las paredes del laberinto. 
flotantes. Resumiendo, Extravia es una historia con una interfaz muy extraña, pero al mismo tiempo muy útil para encerrar un sentido concreto. Este laberinto atípico resulta ser una interfaz hecha para una historia concreta.

\section{El papel líquido: un poema que "requiere" nuestra presencia pero nos salpica}

El último ejemplo analizado aquí será el de El papel líquido, uno de los dos poemas del ciclo Poemas electromagnéticos. Como en muchos de sus trabajos, Aburto Zolezzi olvida las interfaces características de lo electrónico y nos propone un texto que no usa ningún tipo de pantalla. Por eso, a primera vista y en contra de las declaraciones del autor, El papel líquido parece más bien una obra concreta y no un poema electrónico (aunque tuvo su estreno en el festival de la literatura electrónica $\left.{ }^{16}\right)$.

Se trata de un texto escrito (no en versos, sino en círculos, como se ve en la foto - ilustración 2) en un paño blanco cubierto de un fluido no newtoniano, también blanco, para tapar las letras. El fluido se mueve y va descubriendo el poema, pero lo hace solo cuando recibe unos impulsos electromagnéticos emitidos por el bloque en el que está dispuesto el paño con el escrito. Y esta emisión la puede provocar únicamente la presencia del lector. Eso significa que si no hay nadie cerca del texto - no pasa nada, pero cuando alguien se acerca - el "papel líquido” empieza a moverse como si entrara en ebullición y así aparecen partes del poema. Si alguien está muy cerca del texto, puede leerlo todo, pero estar muy cerca del poema significa exponerse al peligro de ser salpicado por lo escrito (por culpa de la materia "hirviente"). Es decir, que en el momento de leer se crea, entre el lector y el texto, una relación muy íntima que puede dejar huellas visibles. La lectura es - como en el pensamiento de Hans-Georg Gadamer - un encuentro de dos "horizontes": uno del lector y el otro del texto mismo.

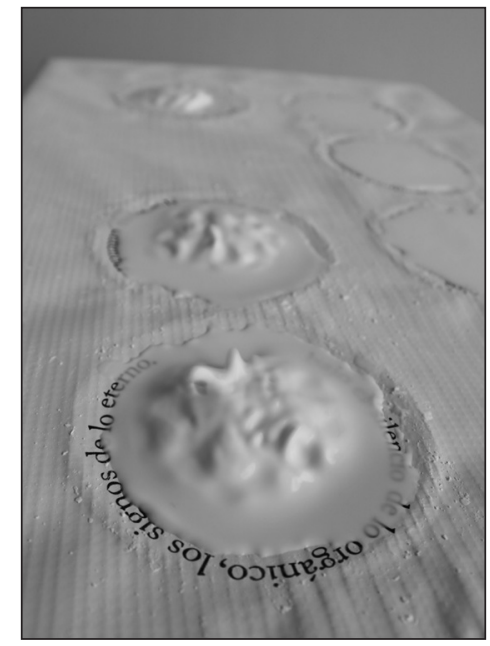

Ilustración 2.

16 En la exposición que acompañaba el congreso anual de la Organización de la Literartura Electrónica (Electronic Literarture Organization) en Victoria (Canada) en junio 2016. 
Esta vez, como en todos los ejemplos analizados anteriormente, el acto de la lectura tan no-típica fue ideado por el autor hasta el más mínimo detalle. La manera de leer la obra ayuda o incluso permite entenderla. El sentido del poema está co-creado (no solo fortalecido) por lo que hace el lector, quien experimenta lo reflejado en el texto. El papel líquido describe el encuentro entre el texto y el lector que tiene el coraje de romper los hábitos y dejarse ensuciar por la lectura, es el que "sabe entender":

Río que hierve de tinta

bajo de falso vacío en blanco

esperando saltar, romper y salpicar

hasta llenar de manchas los ojos

de aquellos que sepan entender

bajo el silencio de lo orgánico, los signos del eterno ${ }^{17}$.

El encuentro descrito no podrá tener lugar si no se siente lo que dice el poema, creado como un evento, no como un artefacto literario (recordando palabras de Katherine Hayles; 2006). El papel líquido es un texto para experimentar que no se revela y no existe sin la presencia corporal de su lector. Por tanto, es otro ejemplo de la búsqueda de una interfaz ideal para la historia única en la obra de Aburto Zolezzi.

\section{El autor siempre en busca de una interfaz ideal}

Todos los textos de Aburto Zolezzi tienen una forma, es decir, una interfaz, interesante y significativa. Lo notamos no solamente en su obra artística. El autor es poeta de tiempos modernos y se presenta con una tarjeta personal, que también cuenta con una interfaz muy propia. Dicha tarjeta a primera vista parece muy tradicional, pero no solo nos informa de los datos personales del poeta y nos da una idea general de su obra (uno de sus poemas está impreso en ella). La podemos mirar también como un manifiesto artístico de Zolezzi: es una tarjeta interactiva, aunque hecha de papel; una tarjeta de interfaz no invisible. Permite a la persona que la coge cambiarla con un simple gesto de doblarla (incluso la forma de la tarjeta invita a hacerlo). Podemos doblarla de distintas maneras, obteniendo como resultado diversos poemas, poemas que gracias a la actividad del lector están todo el tiempo sujetos a modificaciones, no tienen su forma definitiva. Nos introducen perfectamente en el mundo de la poesía de Aburto Zolezzi. Como vemos, el poeta usa la tarjeta que rompe con el popular concepto de la tarjeta personal y que abandona su forma tradicional, aunque a primera vista no lo parece. Al mismo tiempo, el artista inicia un diálogo con la historia y la tradición: en el pasado, las tarjetas personales frecuentemente incluían un detalle personal y/o artístico de su propietario (por ejemplo, una pintura hecha por él, o - ¿por qué no? - un poema, como en este caso). De esta manera, otra vez podemos comprobar que los "juegos" de Aburto Zolezzi tienen su fundamento y explicación. El poeta no solo juega por jugar, lo hace con la intención de buscar las mejores soluciones para sus textos. Con esta tarjeta suya personalizada Aburto Zolezzi se presenta como un artista consciente de la larga tradición del arte y un autor para quien no existen ni reglas ni límites de

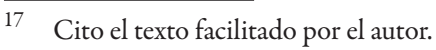


los medios, un artista que busca nuevos caminos para comunicarse, experimentando con la materia, "el cuerpo", la interfaz de sus textos. Lo ilustran los ejemplos mencionados en este artículo.

En conclusión, en todos los trabajos de Aburto Zolezzi analizados, indistintamente del medio o de los medios usados, el texto "necesita" o incluso "exige" una actividad corporal de su lector. En consecuencia, el proceso de leer está totalmente revolucionado, los gestos o actos del lector, su presencia en un lugar concreto o sus movimentos en el espacio real forman parte del acto de la lectura, constituyendo unas condiciones sine qua non para descubrir el sentido de la obra. La experiencia corporal, vinculada con la interactividad de los textos, parece crucial para entender el trabajo o, en algunos casos también incluso para leerlo. La lectura se hace una performance y por eso, interpretando la obra, tenemos que interpretar también las "actividades" del lector (Simanowski 2009).

El abandono de las formas e interfaces más tradicionales que observamos en la obra de Aburto Zolezzi ha nacido de la búsqueda de nuevos medios de expresión y del deseo de renovar la comunicación literaria y de crear unas obras que atraigan y absorban más a lectores modernos (en cuyas vidas se elige entre leer, jugar a videojuegos o ver películas, también interactivas). Pero no era experimentar por experimentar o simple búsqueda de la forma más atractiva. La lectura de Aburto Zolezzi ilustra lo que constituye la base para las teorías del materialismo textual: el hecho de que cada historia pueda "pedir" una interfaz que sea la mejor para ella, que sea la encarnación perfecta del sentido del texto, un "cuerpo" de la historia que no se distingue de ella mísma. Puede ser que esta sea una interfaz invisible (como lo era en muchos ejemplos). Pero este no es el caso de las historias que cuenta Aburto Zolezzi. Buscando interfaces para ellas, el artista abre nuevos espacios para el arte de las Letras: un espacio muy sensual, imprevisible, en el cual cada "encuentro" con un texto es una aventura, una experiencia nueva para la cual no tenemos ni indicaciones, ni llaves. Es así porque las interfaces, igual que las historias, no se repiten. Y hay que buscarlas.

AgniesZKa PrZybysZeWska

(iD) https://orcid.org/0000-0002-0821-3030

Katedra Teorii Literatury, Instytut Kultury Wspólczesnej




\section{Bibliografía}

Aburto Zolezzi José (s. a. a), Hiperpoesía ¿El soporte determinará el contenido?, http://www.artedigitalcuba.cult.cu/6salon/pdf/jose\%20aburto\%20peru.pdf [20.05.2018].

— (s. a. b), sin título, http://www.entalpia.pe/entalpia/absurdo/index.htm [7.05.2018].

— (s. a. c), sin título, http://www.entalpia.pe/ [22.05.2018].

— (s. a.d), sin título, http://www.entalpia.pe/entalpia/_coc/despierto.htm [22.05.2018].

Aarseth Espen (1997), Cybertext. Perspectives on Ergodic Literature, The Johns Hopkins UP, Baltimore-London.

Angel Maria, Gibbs Anna (2013), At the Time of Writing: Digital Media, Gesture, and Handwriting, "Electronic Book Review", 30.08., http://www.electronicbookreview.com/thread/ electropoetics/gesture [1.11.2013].

Bazarnik Katarzyna (2016), Liberature. A Book-bound Genre, Jagiellonian UP, Kraków.

Bouchardon Serge (2014), Figures of Gestural Manipulation in Digital Fictions [en:] Analizing Digital Fiction, eds. Bell A., Ensslin A., Rustad H.K., Routledge, New York.

- (2018), Toward Gestural Specificity in Digital Literature, "Electronic Book Review", 12.02.2018, http://electronicbookreview.com/essay/towards-gestural-specificity-in-digitalliterature/ [20.04.2018].

Drucker Johanna (2011), Humanities Approaches to Interface Theory, "Culture Machine”, no 12.

Emerson Lori (2014), Reading Writing Interfaces. From the Digital to the Bookbound, University of Minesota Press, Minneapolis-London.

Ensslin Astrid (2011), From (w)reader to breather: Cybertextual de-intentionalisation in Kate Pullinger et al.'s Breathing Wall [en:] New Narratives: Stories and Storytelling in the Digital Age, eds. Page R., Thomas B., University of Nebraska Press, Lincoln-London.

Hayles Katherine N. (2002), Writing Machines, MIT Press, London-Cambridge.

- (2006), The Time of Digital Poetry: From Object to Event [en:] New Media Poetics: Contexts, Technotexts, and Theories, eds. Morris A., Swiss T., MIT Press, Boston.

Higgins Dick (1987), Pattern Poetry. Guide to Unknown Literature, State University of New York Press, New York.

High Jeremy (2010), Locative Narrative, Literature and Form [en:] Beyond the Screen. Transformations of Literary Structures, Interfaces and Genres, eds. Schäfer J., Gendola P., transcript, Bielefeld.

Levy Steven (2007), Amazon: Reinventing the Book, http://europe.newsweek.com/amazon-reinventing-book-96909? rm=eu [09.11.2015].

Løvlie Anders Sundnes (2009), Poetic Augmented Reality: Place-bound Literature In Locative Media, https://www.duo.uio.no/bitstream/handle/10852/27215/lovlie_poetic_augmented_ reality_free_access_version.pdf ?sequence $=1$ [30.04.2017].

Marecki Piotr (2018), Między kartka a ekranem. Cyfrowe eksperymenty z medium ksiązki w Polsce, Jagiellonian UP, Kraków.

Pressman Jessica (2009), The Aesthetic of Bookishness in Twenty-First-Century Literature, "Michigan Quarterly Review", fall.

Przybyszewska Agnieszka (2015), Liberackość dzieta literackiego, Wydawnictwo UŁ, Łódź.

Rettberg Scott (2019), Combinatory Poetics [en:] idem, Electronic Literature, Polity Press, Cambridge-Medford.

Rypson Piotr (1989), Obraz stowa. Historia poezji wizualnej, Akademia Ruchu, Warszawa. 
- (2002), Piramidy-stońca-labirynty. Poezja wizualna w Polsce od XVI do XVIII wieku, Wydawnictwo Neriton, Warszawa.

Simanowski Roberto (2009), What is and to What End Do We Read Digital Literature? [en:] Literary Art in Digital Performance. Case Studies in New Media Art and Criticism, ed. Ricardo F.J., continuum, New York-London, http://dm.postmediumcritique.org/Book_LiteraryArtInDigitalPerformance.pdf [28.05.2013].

Starre Alexander (2015), Metamedia. American Book Fictions and Literary Print Culture after Digitalization, University of Iowa Press, Iowa City.

Strehovec Janez (2014), The E-Literary Text as an Instrument and Ride. Novel Forms of Digital Literature and the Expanded Concept of Reading [en:] New Literary Hybrids in the Age of Multimedia Expression: Crossing Borders, Crossing Genres, ed. Cornis-Pope M., Virginia Commonwealth University, Amsterdam/Philadelphia.

White Glyn (2005), Reading the Graphic Surface. The Presence of the Book in Prose Fiction, Manchester UP, Manchester.

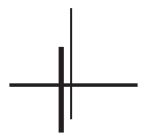

